\title{
Study of Quantum Spin Correlations of Relativistic Electrons
}

\author{
Michał Drągowski*, Jacek Ciborowski \\ University of Warsaw, Faculty of Physics \\ Pasteura 5, 02-093 Warsaw, Poland \\ E-mail: mdragowski@fuw.edu.pl
}

\section{Marek Adamus}

National Centre for Nuclear Research, Świerk

Sottana 7, 05-400 Otwock, Poland

\section{Paweł Caban, Jakub Rembieliński}

University of Lodz, Department of Theoretical Physics

Pomorska 149/153, 90-236 Łódź, Poland

\section{Joachim Enders, Yuliya Fritzsche}

Technische Universität Darmstadt, Institut für Kernphysik

Schlossgartenstr. 9, 64289 Darmstadt, Germany

\section{Marco Dehn, Valery Tioukine}

Johannes Gutenberg-Universität Mainz, Institut für Kernphysik

Johann-Joachim-Becher-Weg 45, 55128 Mainz, Germany

\begin{abstract}
An experiment investigating quantum spin correlations of relativistic electrons is presented. The project aims at the first measurement of the quantum spin correlation function (and the corresponding probabilities) for a pair of relativistic particles with mass. The measurement will be carried out on a pair of electrons in the final state of Møller scattering. The detector consists of two Mott polarimeters, in which the spin projections of both Møller electrons are measured simultaneously.
\end{abstract}

The 18th International Workshop on Polarized Sources, Targets, and Polarimetry, PSTP2019 23-27 September, 2019

Knoxville, Tennessee

${ }^{*}$ Speaker. 


\section{Introduction}

The quantum correlation function is, in general, defined as:

$$
C(A, B)=\sum_{\alpha \beta} \alpha \beta P_{\alpha \beta},
$$

where $P_{\alpha \beta}$ denotes the probability of obtaining $\alpha$ and $\beta$ as a result of measurement of some observables $A$ and $B$, respectively.

Usually, observables $A$ and $B$ are chosen to be the spin projections on some particular directions (denoted $\vec{a}$ and $\vec{b}$ ), measured by two distant observers. For spin $1 / 2$ particles the possible outcomes of each measurement are $\pm \hbar / 2$. It may be assumed for simplicity that spin projections are measured in units of $\hbar / 2$. Then the corresponding spin correlation function $C(\vec{a}, \vec{b})$ yields:

$$
C(\vec{a}, \vec{b})=P_{++}+P_{--}-P_{+-}-P_{-+},
$$

where $P$ with proper indices denote the probability of obtaining a positive $(+)$ or negative $(-)$ spin projection on direction $\vec{a}$ and $\vec{b}$, for the first and second particle, respectively (e.g., $P_{+-}$represents the probability of obtaining positive spin projection for the first particle and negative for the second particle).

This concept dates back to year 1964, when J. Bell published his great paper On the Einstein Podolsky Rosen paradox [1]. In order to prove that quantum mechanics is a nonlocal theory, he proposed the following inequality:

$$
|C(\vec{a}, \vec{b})-C(\vec{a}, \vec{c})| \leq 1+C(\vec{b}, \vec{c}),
$$

which must hold for any theory preserving local realism $(\vec{a}, \vec{b}$ and $\vec{c}$ denote three arbitrary vectors determining the spin projection directions in three correlation experiments). It turns out that it is easy to choose such $\vec{a}, \vec{b}$ and $\vec{c}$ vectors that according to the predictions of quantum mechanics the Bell inequality is violated.

The problem was experimentally settled at the turn of the $70 \mathrm{~s}$ and $80 \mathrm{~s}^{1}$. A. Aspect et al. conducted experiments, which have shown that Bell inequalities are, without any doubt, violated $[2,3]$. Pairs of photons in singlet state were used in these experiments - because of purely technical reasons it is easier to conduct such measurement using photons than with massive particles. It unambiguously proved that the nonlocality of quantum mechanics is not only a feature of the formalism used to describe the reality, but a fundamental property of Nature. It was the ultimate confirmation of the correctness and completeness of quantum mechanics.

A few similar experiments were carried out with massive particles. Table 1 contains information on three experiments, in which the violation of Bell inequalities was tested for spin $1 / 2$ particles with mass (in all three cases protons were used for this purpose).

The expected form of the correlation function for massive particles is different than this obtained within nonrelativistic quantum mechanics, which holds exactly for photons (due to them being massless), as well as in the nonrelativistic approximation for massive particles (e.g., protons

\footnotetext{
${ }^{1}$ some less conclusive experiments were also performed earlier
} 
Table 1: Experiments with protons.

\begin{tabular}{|ll|l|l|}
\hline authors & & year & place \\
\hline Lamehi - Rachti, Mittig & {$[4]$} & 1976 & Saclay (France) \\
Hamieh et al. & {$[5]$} & 2004 & KVI (Holland) \\
Sakai et al. & {$[6]$} & 2006 & RIKEN (Japan) \\
\hline
\end{tabular}

used in the experiments described above, due to their low energy). In the relativistic case, relativistic corrections to the correlation function, dependent on particle momenta, are to be expected.

As an example, a pair of massive spin $1 / 2$ fermions in singlet state may be considered. It is straightforward to show that the correlation function of this system, calculated within nonrelativistic quantum mechanics, has the following form:

$$
C(\vec{a}, \vec{b})=-\vec{a} \cdot \vec{b}
$$

Meanwhile, according to relativistic quantum mechanics [7]:

$$
C(\vec{a}, \vec{b})=-\vec{a} \cdot \vec{b}+\frac{\overrightarrow{k_{1}} \times \overrightarrow{k_{2}}}{m^{2}+k_{1} k_{2}}\left(\vec{a} \times \vec{b}+\frac{\left(\vec{a} \cdot \overrightarrow{k_{1}}\right)\left(\vec{b} \times \overrightarrow{k_{2}}\right)-\left(\vec{b} \cdot \overrightarrow{k_{2}}\right)\left(\vec{a} \times \overrightarrow{k_{1}}\right)}{\left(k_{1}^{0}+m\right)\left(k_{2}^{0}+m\right)}\right),
$$

where $k_{1}$ and $k_{2}$ denote particle four momenta, $m$ their mass. The function consists of a nonrelativistic part identical with equation (1.4) and a relativistic correction dependent on momenta.

Despite this fact, in all of the experiments mentioned in Table 1 the particle energies were insufficient to observe the relativistic correction - their results turned out to be in agreement with the predictions of nonrelativistic quantum mechanics.

\section{Møller scattering}

According to the theoretical predictions, if the relativistic effects are to manifest themselves, the kinetic energy should be of the order of the particle rest mass. Generation of a pure, maximally entangled state in this energy range is a significant problem.

Because of experimental difficulties with preparation of a pure spin state of relativistic particles, the measurement of the correlation function will be performed on a pair of electrons originating from Møller scattering.

The initial state of two electrons (before the scattering) has a product form, as the states of colliding electrons are prepared separately. Since the secondary electrons may be entangled, they do not have, in general, a well determined polarization state. Therefore, in general it is impossible to assign polarization vectors to the electrons after the scattering, because only their mutual polarization state is well defined by the density matrix $\rho^{\text {out }}$ (the explicit form of $\rho^{\text {out }}$ can be found in the paper by Caban et al. [8]). Nevertheless, one can assign mean polarization vectors to both of them.

In order to calculate the mean polarization vector of the $i$-th secondary electron, a partial trace operation over the other one, $j$-th, was performed, yielding a reduced density matrix:

$$
\rho_{i}=\operatorname{Tr}_{j} \rho_{\text {out }}
$$


Next, the mean polarization vector for the $i$-th electron, $\vec{P}_{i}$, was calculated as:

$$
\vec{P}_{i}=\operatorname{Tr}\left(\rho_{i} \cdot \vec{\sigma}\right)
$$

where $\vec{\sigma}=\left(\sigma_{1}, \sigma_{2}, \sigma_{3}\right)$ and $\sigma_{k}$ are the Pauli matrices.

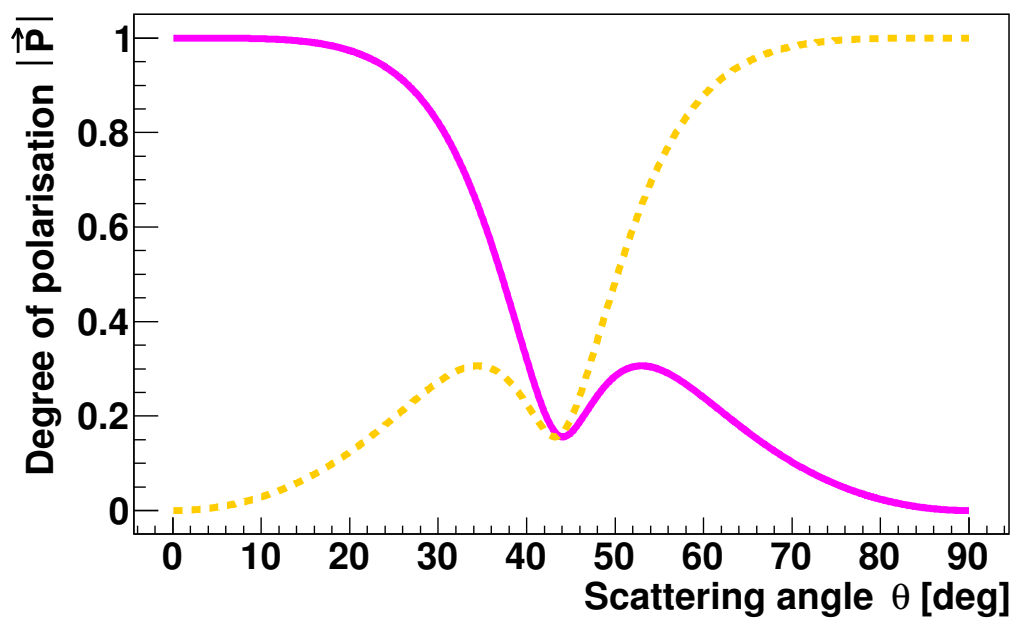

Figure 1: Transfer of polarization from a $100 \mathrm{keV}, 100 \%$ polarized beam electron to the secondary Møller electrons. Lengths of the polarization vectors of each secondary electron are plotted vs. scattering angle $\theta$. Equal sharing occurs for the symmetric scattering configuration $\left(\theta=43.7^{\circ}\right)$.

If, for example, a $100 \%$ polarized beam is scattered off an unpolarized stationary electron, in general both electrons in the final state are partly polarized. This is illustrated in Figure 1. In the symmetric scattering configuration $\left(\theta=43.7^{\circ}\right.$ for a $100 \mathrm{keV}$ incident electron) both outgoing electrons have the same polarization due to the indistinguishability of the particles; however in an asymmetric configuration, in particular for very small scattering angles, the electron with higher energy inherits the majority of the incoming electron polarization.

While the measurement of the mean polarization allows to investigate the average spin state of individual electrons after the scattering, measurement of the spin correlations gives insight into the entanglement production. Both the correlation function and the corresponding probabilities were calculated and thoroughly discussed in a special case of polarized electron beam scattering on an unpolarized target [8], as well as the scattering of two polarized electrons [9].

The correlation function in case of Møller scattering of a polarized electron beam on an unpolarized target, which is the simplest sufficient case that can be realized experimentally, is shown in Figure 2 as a function of beam energy (both $\vec{a}$ and $\vec{b}$ vectors, on which the spins are projected, lie in the Møller scattering plane, and Møller scattering is symmetric with respect to the beam direction). The correlation function exhibits a strongly non-monotonic dependence on beam energy.

Our experimental setup will allow us to perform the measurement for two different configurations of the $\vec{a}$ and $\vec{b}$ vectors: (i) both in the Møller scattering plane, and (ii) at angles equal to $45^{\circ}$ to the Møller scattering plane. The absolute value of the correlation function is small (below 0.1 ) in the range of relativistic energies. Nevertheless, the difference between the nonrelativistic 


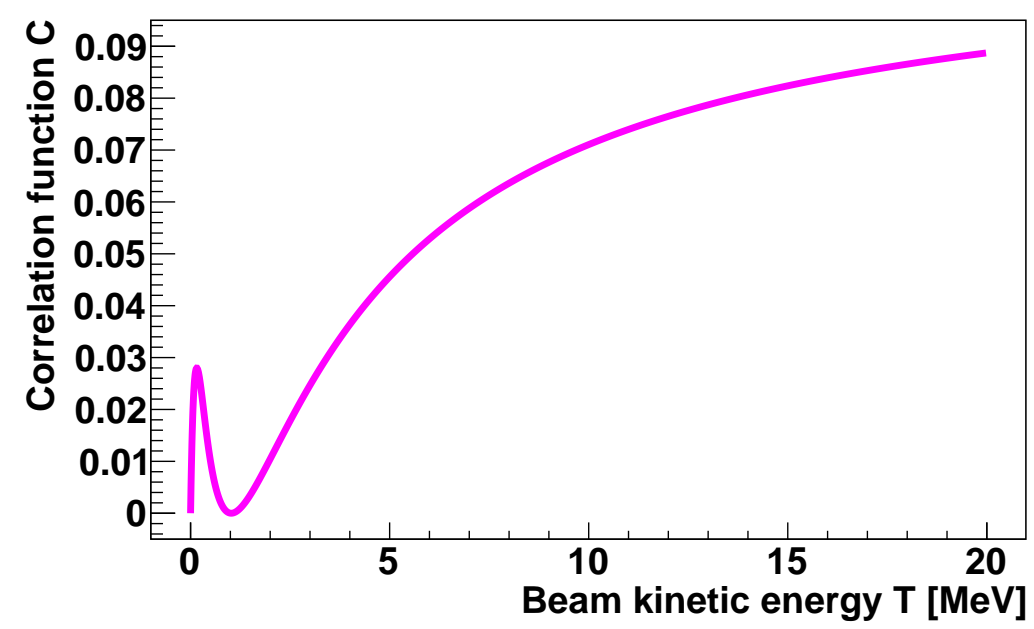

Figure 2: Dependence of the correlation function on beam energy for a pair of electrons originating from Møller scattering (theoretical predictions [8]). The scattering is symmetric with respect to the beam direction and the $\vec{a}$ and $\vec{b}$ vectors (on which the spins are projected) lie in the Møller scattering plane.

and relativistic value (the relativistic correction) can be much larger (of the order of 0.5 in the latter configuration of the $\vec{a}$ and $\vec{b}$ vectors).

It is also worth mentioning that even though the correlation function itself does not depend on beam polarization, the $P_{ \pm \pm}$probabilities do. Therefore, it is important that not only the correlation function is measured in this experiment, but also its corresponding probabilities.

\section{2POL Experiment}

The study of the spin correlations of electron pairs comes down to the measurement of the electron spin projection on a given direction in space. The measurement of the spin projection is challenging in case of relativistic electrons. Fortunately, the applicability of Mott polarimetry was confirmed in the $\mathrm{MeV}$ energy range [10].

The experimental setup consists of two Mott polarimeters, one for each of the two electrons in the final state of Møller scattering. Møller scattering takes place on atomic electrons in a target made of light material (100 $\mu \mathrm{m}$ beryllium). In addition to the standard Mott polarimeter layout, collimators are placed in the arms between the Møller and Mott targets. They accept Møller scattering events close to symmetric kinematical configuration only (in the range $26.75^{\circ} \pm 1.5^{\circ}$ ).

Detection of studied particles is carried out using calorimeters made of scintillating material. Firstly, short reaction time ensures precise timing required to count coincidences between detectors and allows for use of high beam current (which is important in view of low cross sections). Secondly, an approximate measurement of the energy deposited by electrons is possible. Despite low resolution, it allows to distinguish the desired electrons originating from Møller scattering from scattered beam electrons (whose energy is about twice as high).

The experiment comes down to counting the number of coincidences, in which two particles are registered in both Mott polarimeters at the same time. This in turn allows to calculate the 
correlation probabilities and correlation function from the number of coincidences in the detectors recorded in the experiment.

Summarizing, a polarized $3 \mathrm{MeV}$ electron beam is scattered on a stationary (unpolarized) target made of beryllium, each Møller electron passes through one of the main arms leading to a polarimeter, then undergoes Mott scattering on a gold target, passes through an arm selecting backward Mott scattering and deposits its energy in the calorimeter.

The main difficulty in this measurement arises from the extremely low cross sections for studied processes; the double Møller coincidence probability is of the order of $10^{-16}$. A related issue is that of the signal to background ratio, many orders of magnitude lower than in the case of a standard Mott polarimeter. At the present stage there is, in particular, no tracking that could be used to eliminate background. One has to remember that the final result will be disentangled from the number of coincidences, which is the only quantity measured experimentally.

\section{Monte Carlo simulation}

In lack of experimental data a dedicated Monte Carlo simulation [11] was used to optimize the parameters of the experimental setup, including target thicknesses, scattering angles and collimator diameters. It is important to find the optimal compromise between the measured effect and statistical accuracy (e.g., increasing target thickness increases the number of interactions, but also decreases the accuracy of measurement due to multiple scattering and electron depolarization). The criterion, which balances sensitivity to polarization vs. sample size, follows from considering the figure of merit, $\mathscr{F}$, defined by the product:

$$
\mathscr{F}(E, \theta, d) \propto S_{\text {eff }}^{2}(E, \theta, d) N(E, \theta, d),
$$

where $S_{\text {eff }}$ is the effective Sherman function (analyzing power) and $N$ is the total number of scattered electrons recorded in both counters, for energy $E$, scattering angle $\theta$ and target thickness $d$.

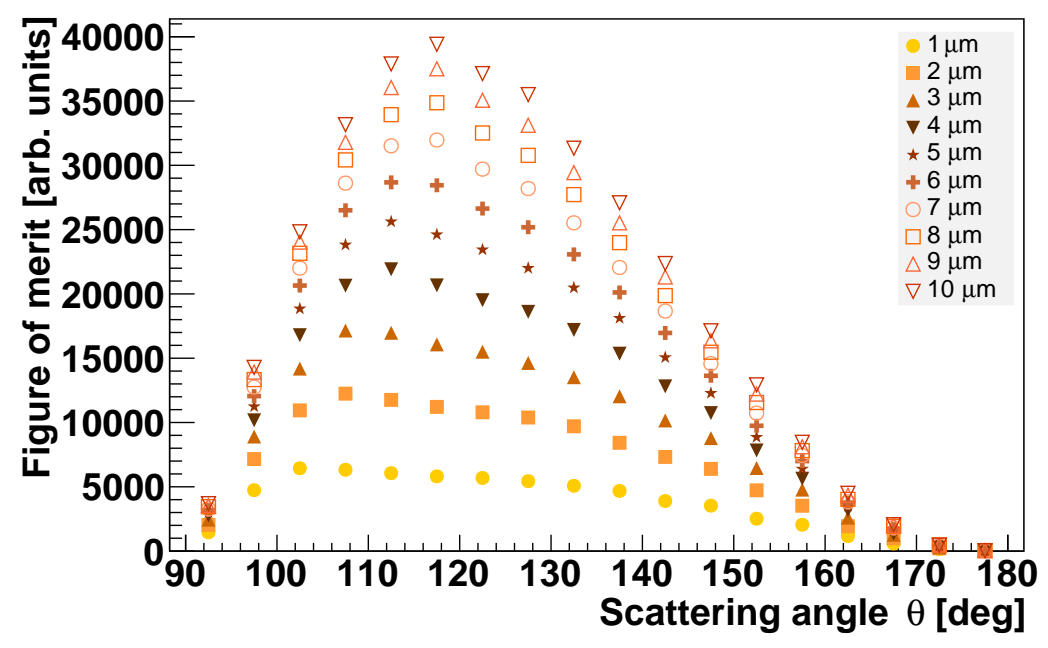

Figure 3: Figure of merit simulated for $2 \mathrm{MeV}$ electrons and different Au target thicknesses. 
As an example, the dependence of the figure of merit on the scattering angle is plotted in Figure 3 for several target thicknesses. Such plots can be used to determine the optimal combination of target thickness and scattering angle for a given beam energy by simply finding the maximum attainable value of the figure of merit.

It is worth pointing out that the approach necessary in case of Mott measurement performed on particles originating from a reaction process is slightly different than in the case of a typical Mott polarimeter operating on beam. In the latter case a target thinner than optimal from the statistics point of view is typically used with the aim of background reduction (the cost of lower statistics is usually neglected in light of the anyway short measurement time). On the contrary, this cannot be afforded in the case of a coincidence measurement of this kind, in which one of the main difficulties arises from scarce statistics caused by the low cross sections mentioned above.

\section{Test measurements}

Test measurements were performed with a $3 \mathrm{MeV}$ polarized electron beam from the injector linac of Mainzer Mikrotron. They were carried out with half of the setup, which can be used as a single polarimeter, allowing to measure the polarization of the beam, as well as the mean polarization of Møller electrons.

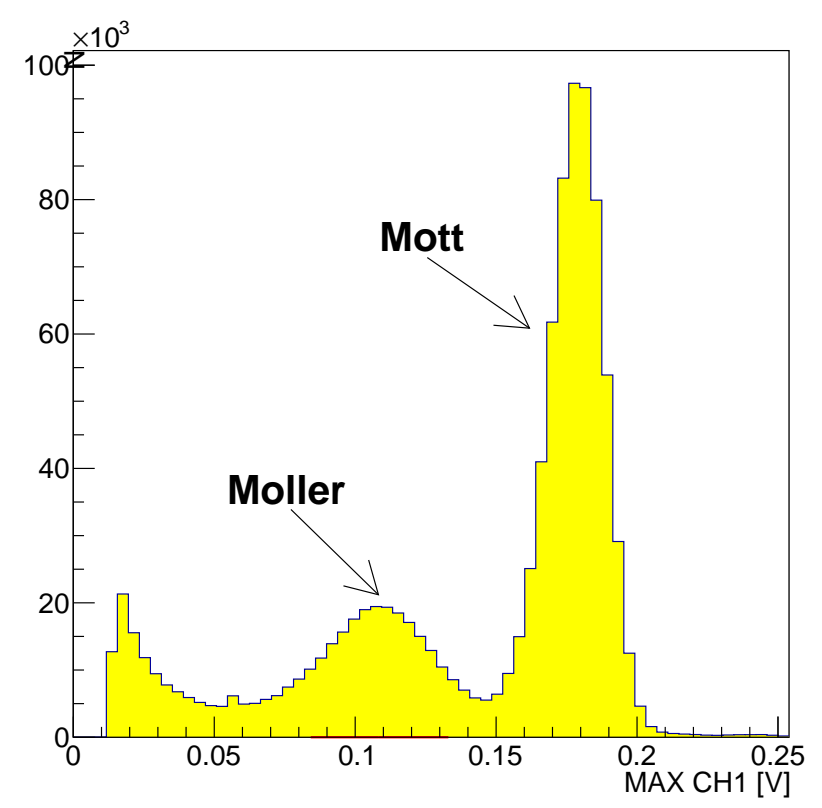

Figure 4: Pulse height spectrum in the auxiliary detector pointing directly at the beryllium target.

The other Mott polarimeter was replaced with a detector pointing directly at the beryllium target (below referred to as the auxiliary detector), allowing to observe the energy spectrum of particles reaching the Mott polarimeter. A typical raw spectrum, taken with a low threshold, is shown in Figure 4. The high, sharp peak corresponds to the beam electrons scattered off the Be target, while the lower peak - to the Møller electrons. The larger width of the Møller peak 


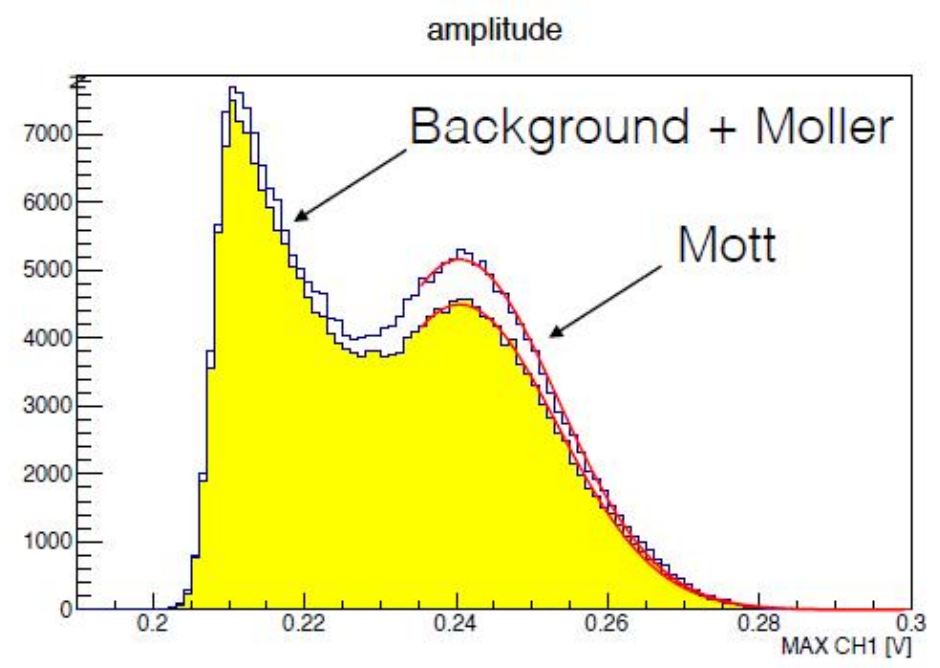

Figure 5: Pulse height spectrum in the polarimeter plotted for opposite beam polarizations separately, allowing to determine the asymmetry.

arises from the angular acceptance of the collimators, which accept asymmetric Møller scattering electrons in a range of energies. The low channel background can be parametrized approximately exponential.

A typical raw spectrum recorded in the polarimeter, taken with a high threshold, is shown in Figure 5 for two opposite beam polarizations. An asymmetry due to the beam polarization is clearly visible; it is equal to $A_{\text {exp }}^{\text {Mott }}=-0.068 \pm 0.004$. Meanwhile, the theoretical prediction is $A_{\text {theory }}^{\text {Mott }}=S P \cos 30^{\circ}=-0.062 \pm 0.002$ (stat.) assuming the Monte Carlo value of the effective Sherman function $S(3 \mathrm{MeV})=-0.0890 \pm 0.0020$ (stat.) and the beam polarization $P=0.81 \pm 0.02$.

The computation of the effective Sherman function takes into account the energy loss of electrons in the Au target. The effect is quite high since the $10 \mu \mathrm{m}$ Au target is relatively thick for electron energies around $3 \mathrm{MeV}$. In order to calculate the effective Sherman function corresponding to the applied event selection, Gaussian smearing, reflecting the detector resolution, was applied to the Monte Carlo energy values, and the effective Sherman function was calculated for events selected from the same part of the spectrum as in the data analysis.

In order to record the Møller electrons in the polarimeter, a coincidence trigger of the auxiliary and polarimeter detectors was used. Event selections acting towards reducing background in the polarimeter can be applied by means of restricting values of two variables: (1) energy (voltage level) in the auxiliary detector and (2) difference of arrival times of the polarimeter and auxiliary detector signals. Figure 6 illustrates the results of event selection procedure on the coincidence data. The middle plot shows the background rejection efficiency achieved in the polarimeter by selecting events with auxiliary detector amplitude in the $\pm 1 \sigma$ range around the Møller peak maximum. The bottom plot shows the final selection results, with the energy cut as above, and time difference $-0.2 \mathrm{~ns}<\Delta t<1 \mathrm{~ns}$, for opposite beam polarizations. The top and middle plots were made from single run data, while the bottom plot combines data from 24 runs, because of scarce statistics.

In light of the fact that the event selection procedure rejects also a significant fraction of signal events, it is better from the statistics point of view to remove background by subtracting the number 


\section{amplitude}
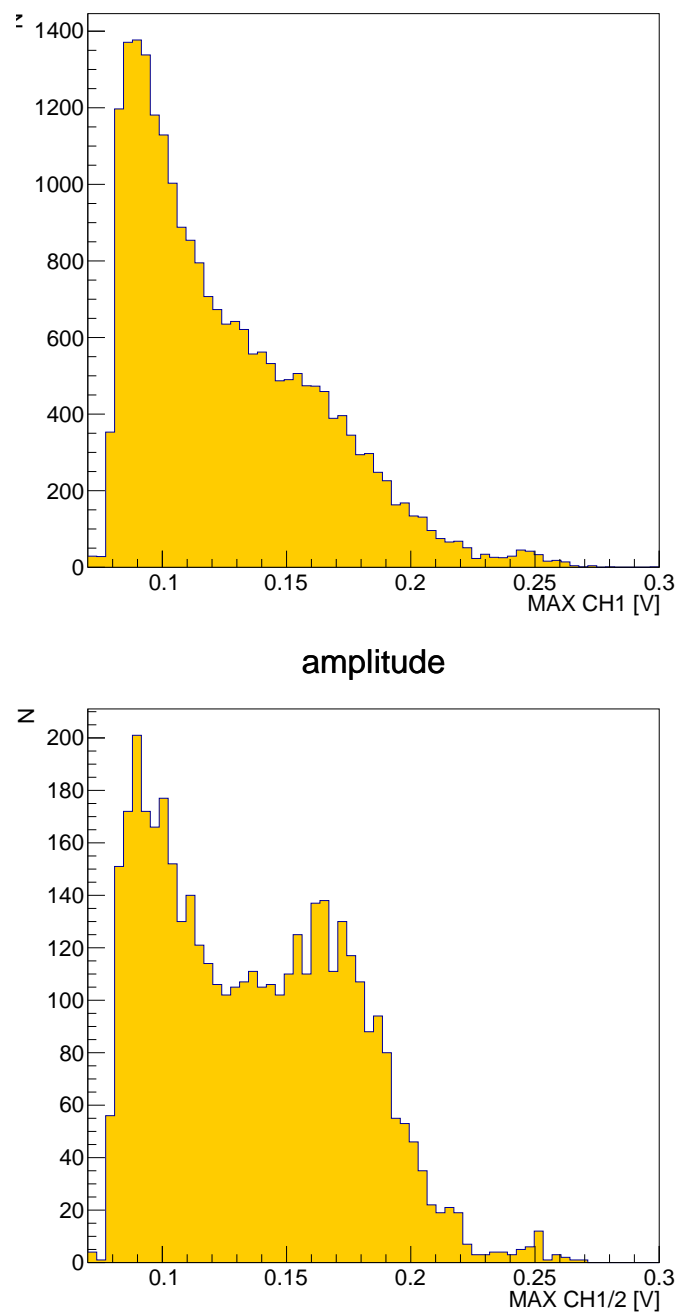

amplitude

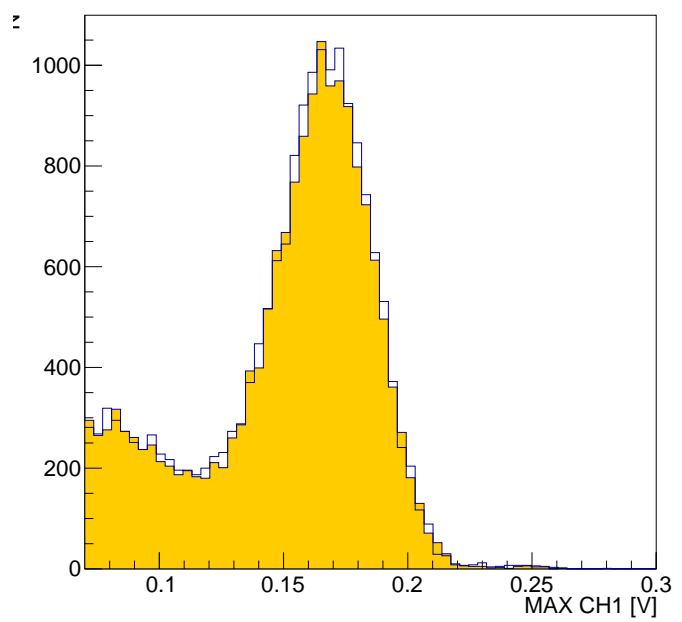

Figure 6: Results of event selection on the amplitude spectra recorded in the polarimeter in coincidence with the auxiliary detector pointing at the beryllium target to record the symmetric Møller scattering events. Top: no selection applied; middle: energy selection in the auxiliary detector; bottom: energy and timing selection. 


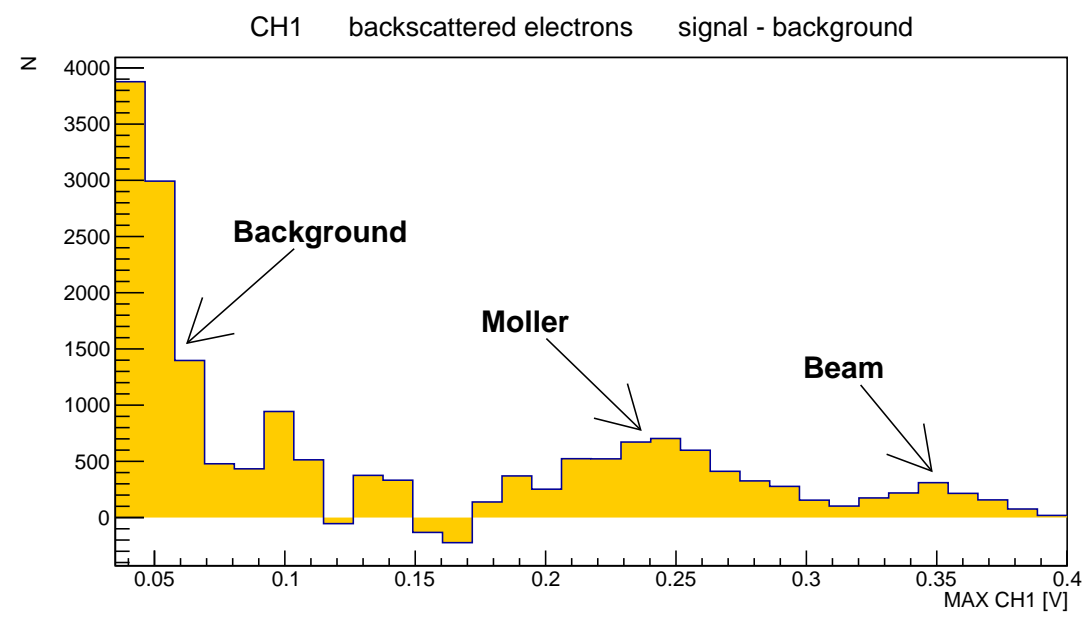

Figure 7: Amplitude spectrum of a detector in the polarimeter after subtracting the data collected with an empty target frame.

of events recorded with an empty target frame from the number of events recorded with the target foil. An example of such analysis is shown in Figure 7. Nevertheless, both methods can be used to cross check the correctness of data analysis.

\section{Summary and conclusions}

The project aims at a first measurement of the spin correlations of relativistic electrons originating from Møller scattering. A prototype of a dedicated polarimeter was successfully tested on beam. The main conclusions from the test measurements are that the principle of operation of the polarimeter has been confirmed; the asymmetry arising due to beam polarization was measured and found in agreement with an independent direct measurement, and the Møller electrons backscattered off the Au target were observed and successfully distinguished from background.

According to the theory, the polarization of symmetric Møller electrons for a $3 \mathrm{MeV}$ beam, with polarization perpendicular to the Møller scattering plane, is equal to $\approx 0.38$ of the beam polarization. The uncertainty of the polarization of Møller electrons measured during the test period is large due to low statistics. This prediction of relativistic quantum mechanics will be verified in a more precise measurement planned for the first half of year 2020.

The expected results regarding the spin correlations are threefold. Firstly, the experiment will allow for a first observation of the relativistic correction to the correlation function. In all of the correlation experiments performed until now the energy of the particles was insufficient to observe relativistic effects. Secondly, the results will be compared to the predictions of relativistic quantum mechanics. The project will be the first attempt to verify the predictions of this theory in the domain of spin correlations and might lead to new discoveries regarding spin observables in relativistic quantum mechanics. Thirdly, by performing the experiment for several beam energies one could experimentally verify the unexpected, non-monotonic dependence of the correlation function on energy (in the configuration shown in Figure 2 the local maximum occurs around $150 \mathrm{keV}$ and the minimum around $1 \mathrm{MeV}$, both measurements are thus technically possible). 


\section{Acknowledgments}

This work was supported by a National Science Centre grant no. 2017/25/N/ST2/00619 (M. Dragowski), and by the German DAAD.

\section{References}

[1] J. S. Bell, Physics 1, 195 (1964)

[2] A. Aspect, P. Grangier and G. Roger, Phys. Rev. Lett. 49, 91 (1982)

[3] A. Aspect, J. Dalibard and G. Roger, Phys. Rev. Lett. 49, 1804 (1982)

[4] M. Lamehi - Rachti, W. Mittig, Phys. Rev. D 14, 2543 (1976)

[5] S. Hamieh et al., J. Phys. G 30, 481 (2004)

[6] H. Sakai et al., Phys. Rev. Lett. 97, 150405 (2006)

[7] P. Caban and J. Rembieliński, Phys. Rev. A 72, 012103 (2005)

[8] P. Caban, J. Rembieliński and M. Włodarczyk, Phys. Rev. A 88, 032116 (2013)

[9] M. Włodarczyk, P. Caban, J. Ciborowski, M. Drągowski, J. Rembieliński, Phys. Rev. A 95, 022103 (2017)

[10] J. Sromicki et al., Phys. Rev. Lett. 82, 57 (1999)

[11] M. Drągowski et al., Nucl. Instr. Meth. Phys. Res. B 389-390, 48 (2016) 